\title{
Intracardiac Flow Visualization Using High-Frame Rate Blood Speckle Tracking Echocardiography: Illustrations from infants with Congenital Heart Disease
}

\author{
Massimiliano Cantinotti ${ }^{1}$, Pietro Marchese ${ }^{1}$, Martin Koestenberger ${ }^{2}$, Raffaele Giordano ${ }^{3}$, \\ Giuseppe Santoro $^{1}$, Nadia Assanta ${ }^{1}$, and Shelby Kutty ${ }^{4}$ \\ ${ }^{1}$ Stabilimento Ospedaliero di Massa Ospedale del Cuore G Pasquinucci \\ ${ }^{2}$ University of Graz \\ ${ }^{3}$ Universita degli Studi di Napoli Federico II \\ ${ }^{4}$ Johns Hopkins Medicine
}

January 6, 2021

\begin{abstract}
We report applications of novel high-frame rate blood speckle tracking (BST) echocardiography in a series of infants with congenital heart disease (CHD). BST echocardiography was highly feasible, reproducible, and fast. High-frame rate BST provided complimentary information to conventional color-Doppler data enhancing the visualization and understanding of anomalous blood trajectories (e.g., shunt direction, regurgitant volumes, and stenotic jets) and vortex formation. High-frame rate BST echocardiography is a new, promising imaging tool that may be helpful for deeper understanding of complex CHD physiology.

Intracardiac Flow Visualization Using High-Frame Rate Blood Speckle Tracking Echocardiography: Illustrations from infants with Congenital Heart Disease

Massimiliano Cantinotti, MD ${ }^{1,2}$; Pietro Marchese, MD ${ }^{1,3}$ Martin Koestenberger, MD4; Raffaele Giordano M.D 5, Giuseppe Santoro M.D. 1; Nadia Assanta, MD¹; Shelby Kutty, MD, MS, PhD 6

1 Stabilimento Ospedaliero di Massa Ospedale del Cuore G Pasquinucci, Pediatric Cardiology and Cardiac Surgery, Massa, Toscana, IT

2 Institute of Clinical Physiology (IFC), National Research Institute (CNR), Pisa, Italy

3 Department of Pediatrics University of Pisa, Italy

${ }^{4}$ Division of Pediatric Cardiology, Department of Pediatrics Medical University Graz, Austria

5 University of Naples "Federico II", Italy,

6 Johns Hopkins Medicine, Pediatrics, Baltimore, MD, USA
\end{abstract}

Running title: Blood Speckle Tracking Echocardiography in CHD

Word Count: 1264 words

Key words : vector flow echocardiography, blood speckle tracking, congenital heart disease

Correspondence $(*)$ : 
Marchese Pietro M.D,

Fondazione CNR-Regione Toscana G. Monasterio

Via Aurelia Sud 5, 54100 Massa (MS)

Tel 3281123173

Email: pitrino91@gmail.com

Fax: none

Authors' conflict of interest disclosure, research founding, employment or leadership: None

\begin{abstract}
We report applications of novel high-frame rate blood speckle tracking (BST) echocardiography in a series of infants with congenital heart disease (CHD). BST echocardiography was highly feasible, reproducible, and fast. High-frame rate BST provided complimentary information to conventional color-Doppler data enhancing the visualization and understanding of anomalous blood trajectories (e.g., shunt direction, regurgitant volumes, and stenotic jets) and vortex formation.
\end{abstract}

High-frame rate BST echocardiography is a new, promising imaging tool that may be helpful for deeper understanding of complex CHD physiology.

High-frame rate blood speckle tracking (BST) echocardiography is a new technique (1-3) based on ultrafast ultrasound imaging for assessing flow patterns. In congenital heart disease (CHD) a variety of complex alterations in blood flow pattern occur, and BST echocardiography may enhance their visualization. High-frame rate BST echocardiography has been technically validated in pediatric patients (3), but clinical applications are to be defined (1-3).

We report a series of infants with normal anatomy and congenital heart disease to demonstrate the benefits of BST echocardiography in this setting.

Images were obtained during outpatient screening or pre-operative examinations at a single Center (Fondazione CNR-Regione Toscana G. Monasterio, Massa, Italy) from a Vivid-TM-E95 ultrasound system (GE Healthcare) with conventional $6 \mathrm{~S}$ and $12 \mathrm{~S}$ phased-array probes, using standard projections and protocols. The BST movies were acquired at frame rates ranging from Clips of 2 cardiac cycles were stored using medium $(66 \mathrm{~cm} / \mathrm{s})$, to low $(53 \mathrm{~cm} / \mathrm{s})$ Nyquist limits.

Images were collected only in quiet and cooperative children. Infants could bottle feed during the examinations. No child was sedated. Approval for this study was obtained from the Local Ethics Committee (Study "Bet2" N.395). Parents or legal guardians of all the children were informed and accepted to participate in the study by signing a written consent.

Case 1: A 2-day old male neonate with body weight $2.85 \mathrm{~kg}$ is presented. Parasternal Long axis (PLAX) view shows normal laminar flow across the aortic valve and aortic root (Figure 1a; Video 1). Modified long axis and short axis views show normal laminar flow across the pulmonary valve and arteries (Figure 1b and Figure 1c; Video 1). A suprasternal view shows normal laminar flow in the ascending aorta and in the aortic arch (Figure 1d; Video 1).

Case 2: This is a 2-day old male neonate with body weight $3.2 \mathrm{~kg}$ and cardiac diagnosis of d-transposition of the great arteries, restrictive patent foramen ovale (PFO), and subaortic ventricular septal defect (VSD). In subcostal view, the PFO with restrictive left-to right shunt can be visualized (Figure 2a). The five-chamber view at different times of the cardiac cycle shows: the RV to PA shunt in systole (Figure 2c), and the shunt from the right ventricle (RV) to the left ventricle (LV) (Figure $2 \mathrm{~d}$ ) in diastole. This is well appreciated by blood speckle tracking (BST). The parallel circulations - RV to aorta (AO) and LV to PA are clearly appreciated in a modified parasternal view (Figure 2 e, Video 2). The high velocity vortical flow across the patent ductus arteriosus is visible in the short axis view (Figure $2 \mathrm{~b}$ and Video 2). 
Case 3: Echocardiogram from a 5-day old male neonate with body weight $3.3 \mathrm{~kg}$ and cardiac diagnosis of severe aortic stenosis (AS). The vortical flow across the aortic valve with vortex formation at the valve, and its propagation above the valve and in ascending aorta (Asc Ao) is best seen in parasternal view (Figures $3 \mathrm{a}$ and 3b, Video 3). On suprasternal view (Figure 3c, Video 3), vortical propagation across the entire aortic arch is seen (Ao ann = aortic annulus). High velocity Doppler (peak velocity $4.3 \mathrm{~m} / \mathrm{s}$, peak gradient $72 \mathrm{mmHg}$ ) through the stenotic aortic valve is seen in 3d. Abdominal aortic flow is blunted and of low pulsatility (3e).

Case 4: This is a 4-day old female neonate with body weight $2.8 \mathrm{~kg}$ and cardiac diagnosis of tetralogy of Fallot (TF) and absent pulmonary valve. In a modified apical 4 chamber view, the systolic right to left (Figure 4a) and diastolic left to right (Figure 4b) flow across the VSD can be appreciated. In Video 4, the shunt is demonstrated through the entire cardiac cycle. Modified right ventricular outflow tract view (RVOT) shows the vortical flow across the rudimental pulmonary valve with vortex formation (Figure 4c, Video 4), and vortex propagation into the dilated pulmonary artery (Figure 4c, Video 4). The diastolic free regurgitation across the pulmonary valve (Figure 4d, Video 4), and the late diastolic vortex formation in the RVOT (Figure 4e, Video 4) are also seen.

Case 5: Echocardiogram from a 4-day old male neonate with body weight $3.1 \mathrm{~kg}$, and severe pulmonary stenosis after pulmonary balloon valvuloplasty. The right to left shunt across the PFO (Figure 5a, Video 5) is seen in the subcostal view. In the 4-chamber view, severe tricuspid valve regurgitation is visualized (Figure 5b, Video 5). The short axis view shows (Video 5) vortex propagation into the pulmonary arteries during the cardiac cycle. Figure 5c shows the vortex at the level of the pulmonary valve (beginning of the systole), the vortex propagating into the main pulmonary artery (mid-systole, $5 \mathrm{~d}$ ), and subsequently into pulmonary artery branches (late systole and early diastole, 5e). Left-to-right shunt from the ductus arteriosus into the main PA is seen, as well as the diastolic regurgitant flow (5f and $5 \mathrm{~g}$ ).

Case 6: This is a 5-day old female

Case 7: Echocardiogram from a 3-day old male neonate with body weight $3.0 \mathrm{~kg}$ and diagnosis of aortic coarctation (ACo). On suprasternal imaging, the vortical flow across the stenotic isthmus below the left subclavian artery (LSA) is seen (Figure 7a, Video 7); there is continuous diastolic flow (Figure 7d; Video 7). Diastolic run-off (Figure 7b) and blunted, low velocity flow in the abdominal aorta (Figure 7c).

Case 8: Echocardiogram in a 1-day old male neonate with body weight $3.4 \mathrm{~kg}$ and diagnosis of total intracardiac anomalous pulmonary venous return. The subcostal view shows pulmonary venous drainage into the coronary sinus (CS) and to the right atrium (RA). The right to left shunt through a large atrial septal defect (ASD) is seen. The trajectory of pulmonary blood from coronary sinus to the right atrium and to the right ventricle (RV), and partly back to the left atrium (LA) through the ASD can be appreciated in subcostal views at slightly different angulations (Figure 8a and $8 \mathrm{~b}$ and Video 8), and in the 4-chamber view (Figure 8c and 8d, Video 8). In the subcostal view simultaneous BST images and 2D images without color are shown.

In the cases we presented high-frame rate BST echocardiography supplemented conventional color-Doppler data, and allowed for a more detailed evaluation of vortex formation and anomalous blood trajectories. High-frame rate BST echocardiography facilitated improved understanding of shunt direction, regurgitant volumes, and stenotic jets vortices with their propagation into the vessels.

High quality high-frame rate BST loop acquisition at neonatal heart rates were feasible (in $100 \%$ of the cases) and fast as to conventional color Doppler. The image post-processing was rapid, requiring not more than 30-60 seconds in each case.

In conclusion, high-frame rate BST echocardiography is a useful tool for enhancing understanding of CHD physiology and could also serve for teaching purposes.

\section{References}

1. Villeman O, Baranger J, Friedberg MK, et al. Ultrafast Ultrasound Imaging in Pediatric and Adult 
Cardiology: Techniques, Applications, and Perspectives JACC Cardiovasc Imaging 2020;13:1771-1791.

2. Thomas Collins R, Lughlin ME, Lang SM, et al. Real-Time Transthoracic Vector Flow Imaging of the Heart in Pediatric Patients. Prog Pediatr Cardiol. 2019 Jun; 53: 28-36.

3. Nyrnes SA, Fadnes S, Wigen MS, Mertens L, Lovstakken L. Blood Speckle-Tracking Based on HighFrame Rate Ultrasound Imaging in Pediatric Cardiology. J Am Soc Echocardiogr. 2020; 33:493-503.e5.

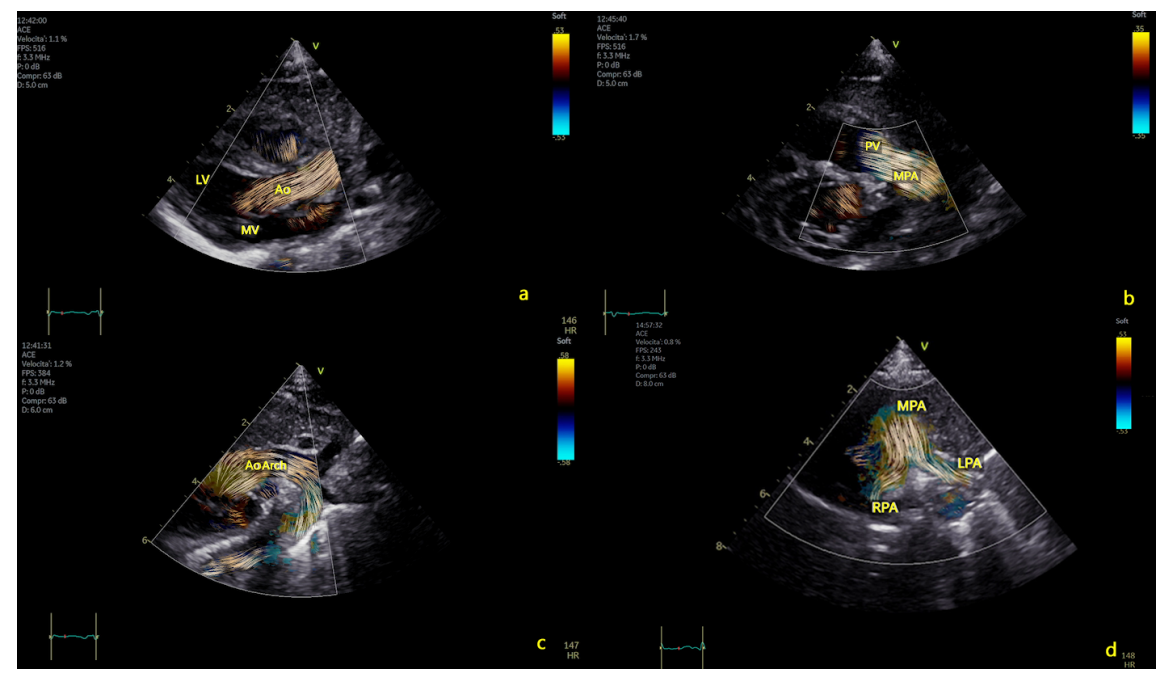



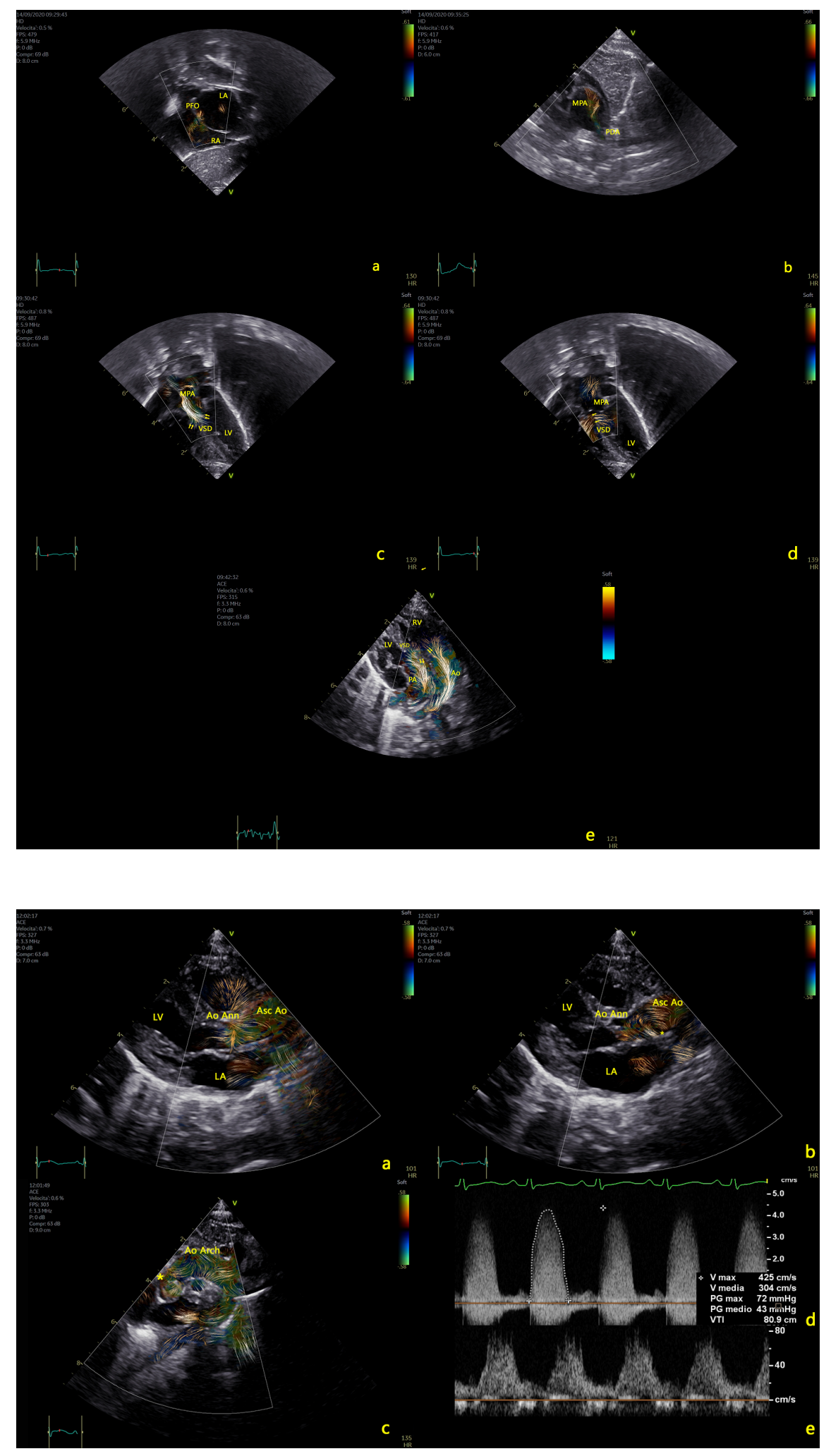

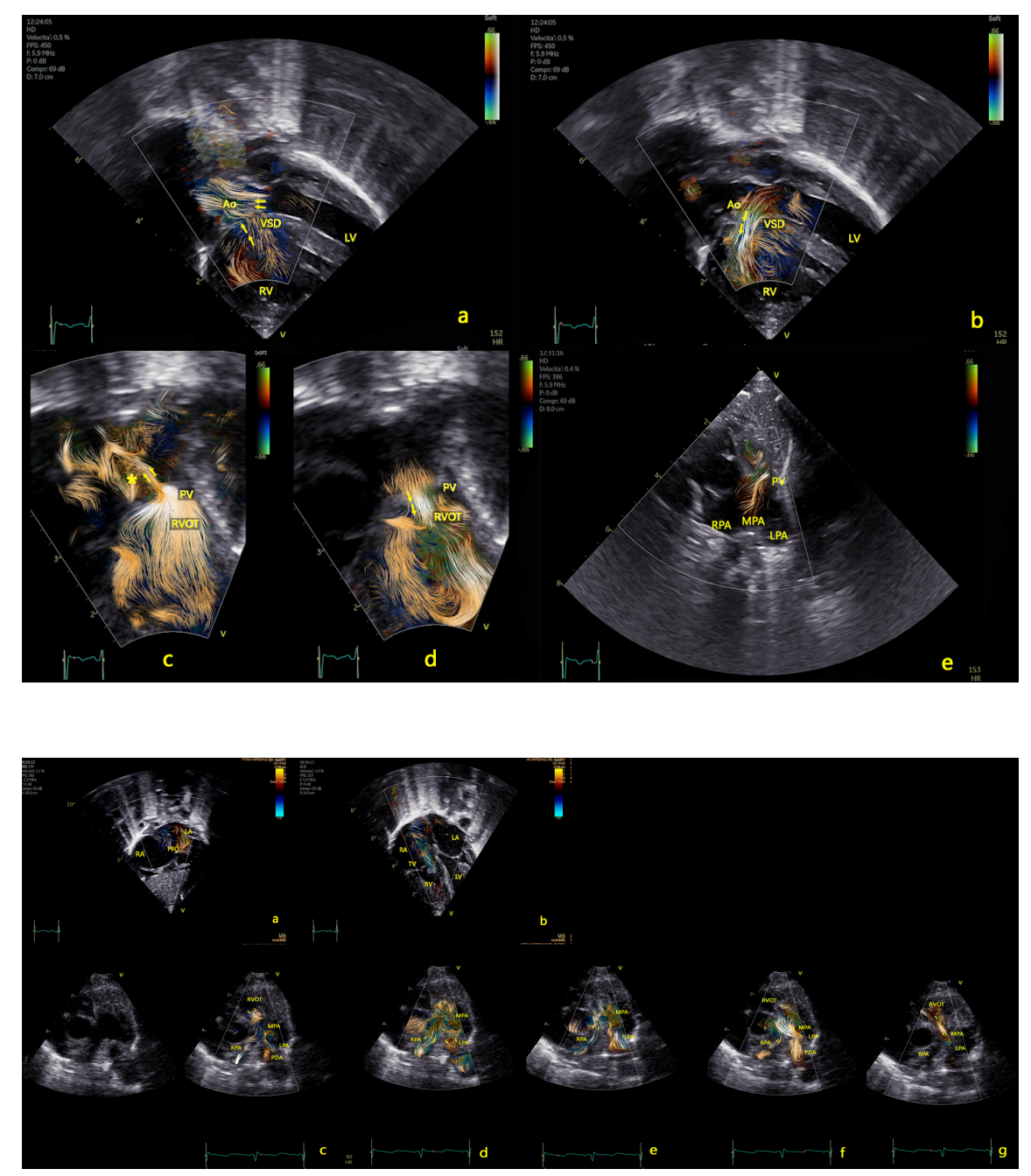


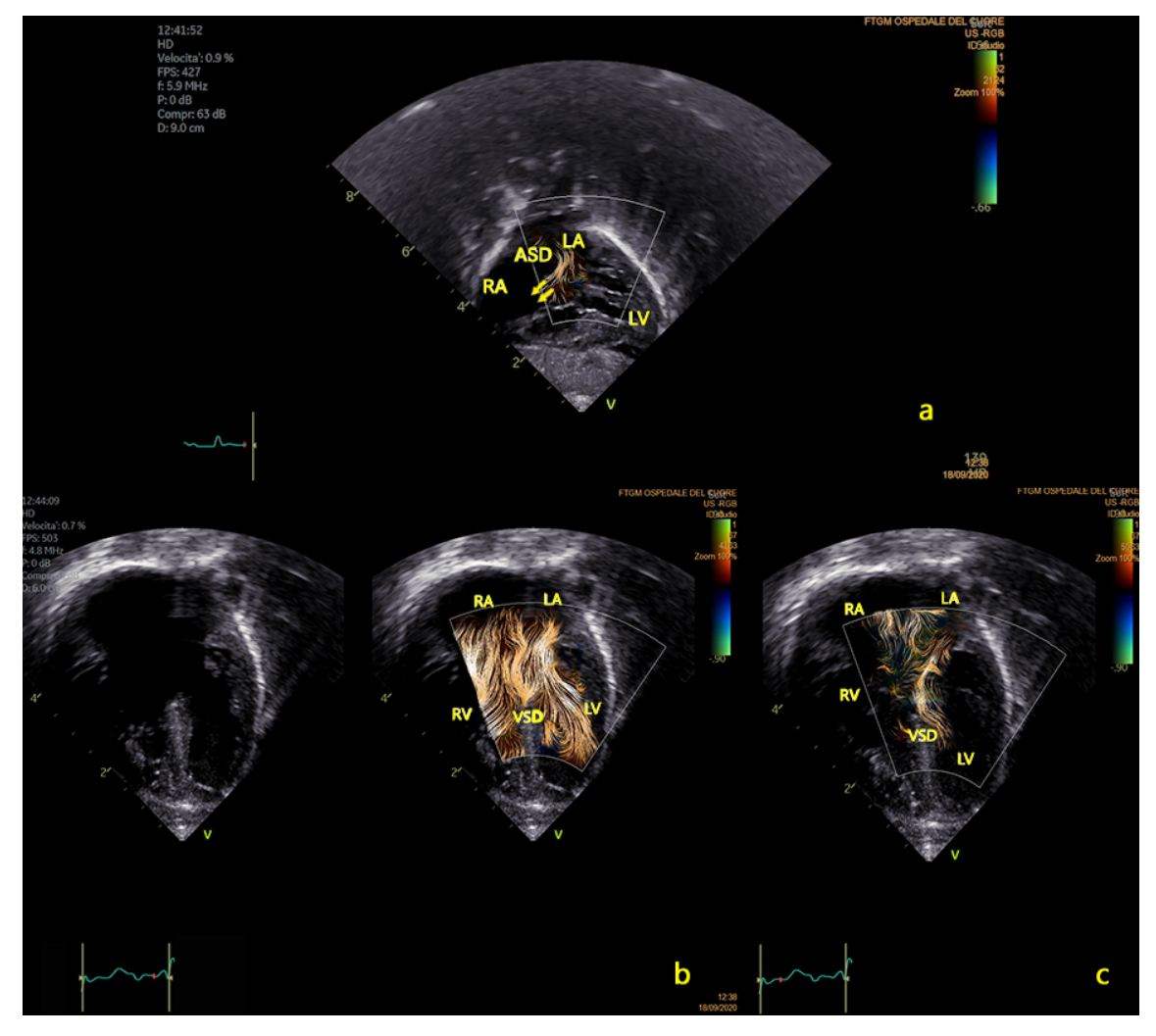




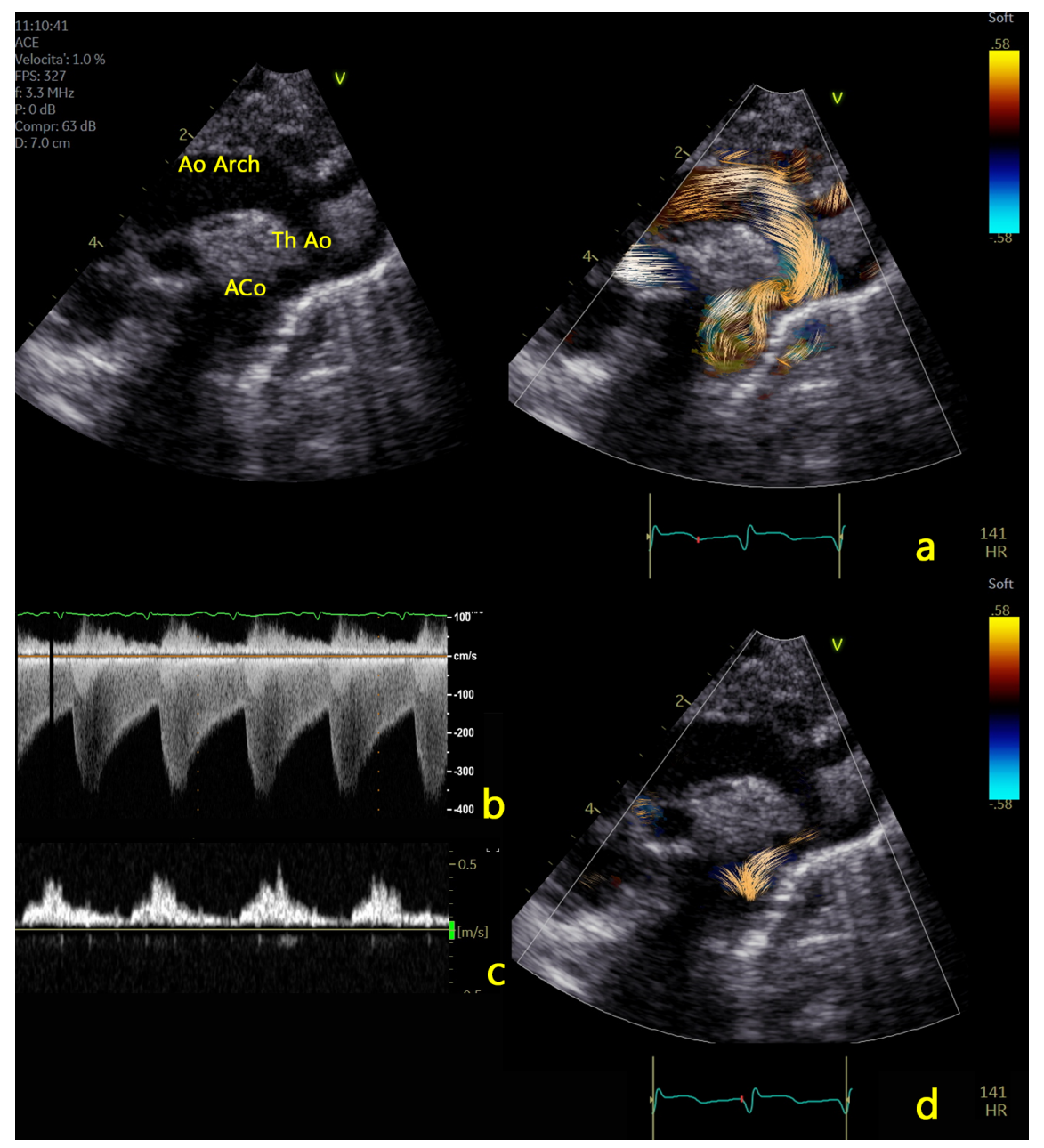




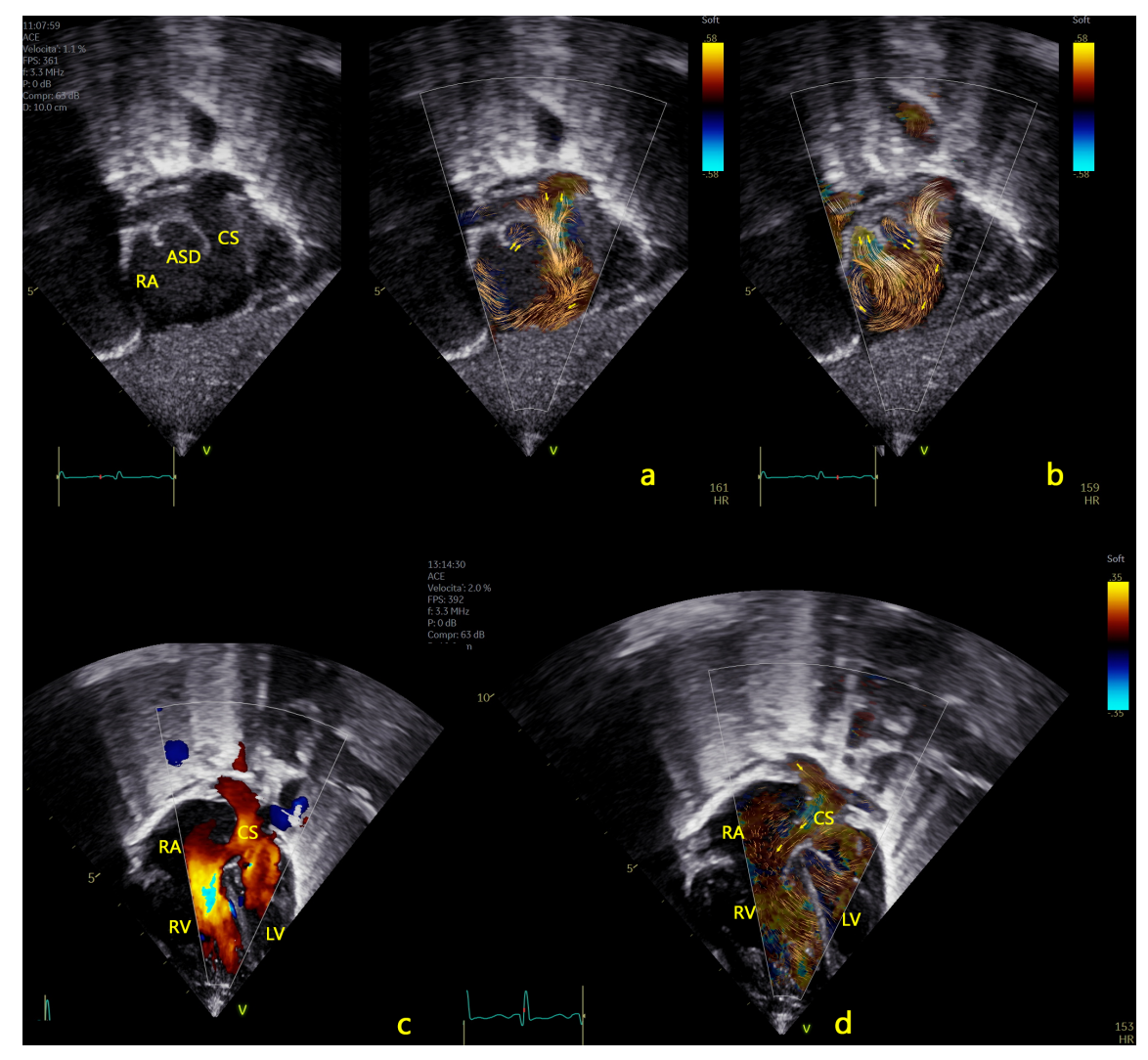

